\title{
Mortalidad Infantil en las comunas de la Región Metropolitana, periodo 2005-2014
}

\author{
Infant Mortality in the Metropolitan Region, Chile 2005-2014
}

\author{
Fernando González ${ }^{\mathrm{a}, \mathrm{b}}$, Tania Alfaro ${ }^{\mathrm{c}}$, Olivia Horna-Campos ${ }^{\mathrm{c}}$ \\ aDepartamento de Salud Pública, Facultad de Medicina, Universidad de los Andes \\ 'Departamento de Pediatría y Cirugía Infantil Sur, Facultad de Medicina, Universidad de Chile \\ 'Escuela de Salud Pública, Facultad de Medicina, Universidad de Chile
}

Recibido: 17 de mayo de 2019; Aceptado: 28 de septiembre de 2019

\section{¿Qué se sabe del tema que trata este estudio?}

Se sabe que la mortalidad infantil a nivel nacional es de las más bajas en Latinoamérica, sin embargo no ha disminuido significativamente en el último decenio ni se han logrado reducir las brechas existentes dentro del territorio, como es a nivel de las comunas.

\begin{abstract}
¿Qué aporta este estudio a lo ya conocido?
Aporta al conocimiento de las brechas de inequidad entre comunas de mayor y menor mortalidad infantil y sus subcomponentes, profundizando en la importancia de caracterizar las diferencias a nivel territorial para desarrollar políticas públicas acordes a las necesidades locales
\end{abstract}

\section{Resumen}

Introducción: La mortalidad infantil depende del nivel de desarrollo económico, social y cultural del área de residencia. Objetivo: Describir las tasas de mortalidad infantil (TMI) y mortalidad infantil tardía (TMIT) de las comunas de la Región Metropolitana (RM) y evaluar su tendencia en el periodo 2005-2014. Material y Método: Estudio ecológico que describe las tasas en las 52 comunas de la Región Metropolitana (RM). Para el análisis se construyó la TMI y TMIT para cada año y comuna y se compararon mediante riesgo atribuible poblacional (RAP), Riesgo atribuible porcentual (RAP\%) y razón de tasas (RT). Las tendencias se evaluaron con el modelo Prais-Winsten. Se consideró una tendencia estadísticamente significativa un valor $p<0,05$. Resultados: La comuna de Independencia mostró la TMI y TMIT más altas con 12,7 y 4,05 por mil RNV respectivamente; 1,75 y 2,05 veces comparado con la TMI y TMIT de la RM. Las tasas más bajas se observaron en Las Condes (TMI) y en Vitacura (TMIT). La tendencia de la TMI respecto al 2005 aumentó en Lo Barnechea, Lo Espejo y Recoleta y disminuyó en Las Condes, Macul, Pudahuel y San Bernardo. La TMIT disminuyó en Peñalolén, Puente Alto, Las Condes, Providencia, San Bernardo, Macul, Pudahuel, Talagante, Pedro Aguirre Cerda y Quilicura y aumentó en Peñaflor. Conclusión: La TMI y la TMIT regional ocultan el leve incremento de las tasas y la persistencia de la heterogeneidad entre las comunas, lo que obliga a explorar las causas de estas inequidades en estudios analíticos a futuro.
Palabras clave:

Mortalidad infantil; mortalidad infantil

tardía; residencia; inequidad; Chile 


\begin{abstract}
Introduction: Infant mortality depends on the economic, social, and cultural level of development of the place of residence. Objective: To describe the infant mortality rates (IMR) and the late infant mortality rates (LIMR) of the Metropolitan Region (MR) communes and to evaluate their trend between 2005 and 2014. Material and Method: Ecological study that describes the rates of the 52 communes of the MR. For the analysis, the IMR and LIMR were elaborated for each year and commune and were compared using population attributable risk (PAR), attributable risk percent (AR\%), and rate ratio (RR). Trends were analyzed through the Prais-Winsten model. A value $\mathrm{p}<0.05$ was considered a statistically significant trend. Results: The commune 'Independencia' presented the highest IMR and LIMR with 12.7 and 4.05 per 1000 live births respectively, 1.75 and 2.05 times more compared with the IMR and LIMR of the MR. The commune 'Las Condes' and 'Vitacura' presented the lowest IMR and LIMR respectively. The IMR trend regarding 2005 increased in Lo Barnechea, Lo Espejo, and Recoleta, and decreased in Las Condes, Macul, Pudahuel and San Bernardo. The LIMR decreased in Peñalolén, Puente Alto, Las Condes, Providencia, San Bernardo, Macul, Pudahuel, Talagante, Pedro Aguirre Cerda, and Quilicura, and increased in Peñaflor. Conclusion: The regional IMR and LIMR hide the slight increase in rates and the persistence of heterogeneity among communes. This forces us to explore the causes of these inequities through future analytical studies.
\end{abstract}

Keywords:

Infant Mortality;

Late Infant Mortality; residence;

inequity;

Chile

\section{Introducción}

La tasa de mortalidad infantil (TMI) es un indicador que representa fuertemente el nivel de salud de una población ${ }^{1-3}$. Una tasa elevada refleja un importante porcentaje de muertes evitables y prematuras, que pueden prevenirse mediante acciones individuales $y$ colectivas orientadas a influir sobre los determinantes sociales y económicos de la salud ${ }^{4-9}$. Uno de los primeros estudios de segregación social, realizado en Nueva York en 1948, describe las diferencias entre población afroamericana y caucásica ${ }^{10}$. Las TMI y fetal estaban directamente relacionadas con la proporción de población afroamericana que vivía en el barrio, correspondiendo al primer reporte de segregación residencial. Otro artículo demostró que la privación social de una determinada área geográfica, medida por el índice de privación de Townsend, -nivel de desempleo, vivienda y hacinamiento-, está fuertemente asociado a TMI, encontrando un gradiente socioeconómico en que el $20 \%$ de la población de menor nivel socioeconómico presenta una mortalidad infantil que varía entre un $21 \%$ y un $55 \%$ mayor que la del mejor nivel socioeconómico ${ }^{11}$. Estudios realizados en países de América Latina y el Caribe han demostrado que el aumento en el acceso de las mujeres a la educación, y en la cobertura de la vacunación, entre otros factores, disminuyeron las TMI de la región, por medio de la disminución del gradiente social ${ }^{12-14}$.

En Chile la TMI en el 2015 fue 6,9 por cada mil nacidos vivos (NV), lo que implicó una disminución de 0,1 respecto al año 2014. Si bien es una de las tasas más bajas de Latinoamérica, se ha demostrado que su distribución es heterogénea entre las regiones y comunas del país y depende de las variables socioeconómicas ${ }^{15}$.
Hertel observó que los desfavorecidos en términos socioeconómicos presentan un riesgo significativamente mayor de TMI por enfermedades infecciosas y trauma durante el primer mes de vida ${ }^{16}$. La Tasa de Mortalidad infantil tardía (TMIT) o postneonatal es un indicador interesante de observar ya que representa con una mayor sensibilidad el impacto de los determinantes sociales de la salud sobre los grupos poblacionales ${ }^{16}$.

El área de residencia es un importante determinante que modela las condiciones en que viven las personas, constituyendo un mayor o menor "riesgo" de enfermar o morir ${ }^{17,18}$. El objetivo es describir las TMI de las comunas de la Región Metropolitana (RM) y evaluar su tendencia en el periodo 2005-2014.

\section{Material y Método}

Estudio ecológico descriptivo que explora la distribución espacial de la TMI y TMIT en las 52 comunas de las 6 provincias de la Región Metropolitana (RM): Santiago, Cordillera, Chacabuco, Maipo, Melipilla, Talagante. El periodo analizado es de 10 años, entre el 2005 y el 2014.

La unidad de análisis es la comuna. La tasa de mortalidad infantil es construida a partir de la suma del total de defunciones de menores de 1 año en cada comuna por cada mil NV dentro de los 10 años revisados. La TMIT por su parte es construida a partir de la suma del total de defunciones de lactantes mayores de 28 días y menores de 1 año en cada comuna por cada mil NV en el mismo período. Estos datos fueron obtenidos del Departamento de Estadísticas e Información de Salud (DEIS) y del Instituto Nacional de Estadísticas (INE).

La comparación de las TMI de las comunas se rea- 
lizó con el estándar regional y con la comuna de menor tasa de las analizadas. Para ello se utilizaron tres indicadores: Riesgo atribuible poblacional (RAP), riesgo atribuible porcentual (RAP\%), y razón de tasas (RT). El RAP se calculó según la fórmula RAP $=($ TMI comunal - TMI referencia), el RAP\% $=(\mathrm{RAP} / \mathrm{TMI}$ comunal $)^{\star} 100$, la RT se calculó según la fórmula RT $=$ (TMI comunal / TMI Referencia). Cuando se usó como referencia la comuna de menor tasa, el RAP y el RAP\% se expresaron como RAPC y RAPC\%, respectivamente.

La tendencia de las TMI por comuna y año se evaluaron con el modelo de Prais-Winsten, con gráficos elaborados en programa STATA 14.0. Se consideró una tendencia al aumento o disminución estadísticamente significativa con un valor $\mathrm{p} \leq 0,05$. La distribución espacial de las TMI en la RM se muestra en un mapa elaborado con el programa R.

\section{Resultados}

\section{Comparación comunal con estándar regional}

Entre el 01 de enero del 2005 y el 31 de diciembre del 2014 en la RM nacieron 989.173 niños y ocurrieron 7.164 defunciones de menores de un año, lo que resulta en una TMI para la década de 7,24 por mil NV. $\mathrm{Al}$ analizar las provincias de la RM, Maipo presentó la mayor TMI la que fue de 9,01 por mil RNV. Las provincias con menor TMI fueron Melipilla y Chacabuco. En la provincia de Santiago, nacieron 729.745 niños y fallecieron 3.674, lo que corresponde a una TMI de 7,10 por mil RNV en el decenio estudiado.

Las comunas en las que se observaron mayores TMI fueron las comunas de San Bernardo e Independencia. Independencia presenta una tasa de 12,7 por mil NV. Su RAP, respecto a la RM es de 5,46 por mil $\mathrm{NV}$, con un RAP\% de $43 \%$ y una RT de 1,75 (tabla 1).

\begin{tabular}{|c|c|c|c|c|c|c|c|c|c|}
\hline Comuna & $\begin{array}{l}\text { Nacimientos } \\
\text { totales }\end{array}$ & $\begin{array}{l}\text { Defunciones } \\
\text { infantiles totales }\end{array}$ & $\begin{array}{c}\text { TMI } \\
\text { (por mil RNV) }\end{array}$ & RAP* & RAP\%* & $\mathrm{RT}^{*}$ & $\mathrm{RAPC}^{\wedge}$ & RAC $\% \wedge$ & $\mathrm{RT}^{\wedge} \wedge$ \\
\hline Santiago & 44.574 & 288 & 6,46 & $-0,78$ & $-12,05$ & 0,89 & 1,58 & 24,46 & 1,32 \\
\hline Cerrillos & 11.711 & 78 & 6,66 & $-0,58$ & $-8,70$ & 0,92 & 1,78 & 26,73 & 1,36 \\
\hline Cerro Navia & 20.607 & 142 & 6,89 & $-0,35$ & $-5,07$ & 0,95 & 2,01 & 29,17 & 1,41 \\
\hline Conchalí & 18.635 & 141 & 7,57 & 0,33 & 4,31 & 1,05 & 2,69 & 35,54 & 1,55 \\
\hline El Bosque & 25.058 & 215 & 8,58 & 1,34 & 15,62 & 1,19 & 3,70 & 43,12 & 1,76 \\
\hline Estación Central & 17.931 & 137 & 7,64 & 0,40 & 5,24 & 1,06 & 2,76 & 36,13 & 1,57 \\
\hline Huechuraba & 15.808 & 117 & 7,40 & 0,16 & 2,18 & 1,02 & 2,52 & 34,05 & 1,52 \\
\hline Independencia*** & 11.657 & 148 & 12,70 & 5,46 & 42,98 & 1,75 & 7,82 & 61,57 & 2,60 \\
\hline La Cisterna & 11.002 & 76 & 6,91 & $-0,33$ & $-4,81$ & 0,95 & 2,03 & 29,38 & 1,42 \\
\hline La Florida & 48.214 & 319 & 6,62 & $-0,62$ & $-9,43$ & 0,91 & 1,74 & 26,28 & 1,36 \\
\hline La Granja & 19.699 & 139 & 7,06 & $-0,18$ & $-2,60$ & 0,97 & 2,18 & 30,88 & 1,45 \\
\hline La Pintana & 34.063 & 230 & 6,75 & $-0,49$ & $-7,22$ & 0,93 & 1,87 & 27,70 & 1,38 \\
\hline La Reina & 9.885 & 60 & 6,07 & $-1,17$ & $-19,28$ & 0,84 & 1,19 & 19,60 & 1,24 \\
\hline Las Condes ** & 36.282 & 177 & 4,88 & $-2,36$ & $-48,41$ & 0,67 & 0,00 & 0,00 & 1,00 \\
\hline Lo Barnechea & 12.826 & 75 & 5,85 & $-1,39$ & $-23,81$ & 0,81 & 0,97 & 16,58 & 1,20 \\
\hline Lo Espejo & 15.934 & 138 & 8,66 & 1,42 & 16,40 & 1,20 & 3,78 & 43,65 & 1,77 \\
\hline Lo Prado & 14.611 & 106 & 7,25 & 0,01 & 0,20 & 1,00 & 2,37 & 32,69 & 1,49 \\
\hline Macul & 15.190 & 107 & 7,04 & $-0,20$ & $-2,78$ & 0,97 & 2,16 & 30,68 & 1,44 \\
\hline Maipú & 70.809 & 511 & 7,22 & $-0,02$ & $-0,32$ & 1,00 & 2,34 & 32,41 & 1,48 \\
\hline Ñuñoa & 24.106 & 143 & 5,93 & $-1,31$ & $-22,05$ & 0,82 & 1,05 & 17,71 & 1,22 \\
\hline Pedro Aguirre Cerda & 14.564 & 111 & 7,62 & 0,38 & 5,01 & 1,05 & 2,74 & 35,96 & 1,56 \\
\hline Peñalolén & 38.315 & 295 & 7,70 & 0,46 & 5,97 & 1,06 & 2,82 & 36,62 & 1,58 \\
\hline Providencia & 17.984 & 109 & 6,06 & $-1,18$ & $-19,45$ & 0,84 & 1,18 & 19,47 & 1,24 \\
\hline Pudahuel & 35.091 & 208 & 5,93 & $-1,31$ & $-22,14$ & 0,82 & 1,05 & 17,71 & 1,22 \\
\hline Quilicura & 32.123 & 259 & 8,06 & 0,82 & 10,20 & 1,11 & 3,18 & 39,45 & 1,65 \\
\hline Quinta Normal & 15.075 & 114 & 7,56 & 0,32 & 4,26 & 1,04 & 2,68 & 35,45 & 1,55 \\
\hline Recoleta & 23.662 & 201 & 8,49 & 1,25 & 14,77 & 1,17 & 3,61 & 42,52 & 1,74 \\
\hline Renca & 22.565 & 164 & 7,27 & 0,03 & 0,38 & 1,00 & 2,39 & 32,87 & 1,49 \\
\hline San Joaquín & 12.100 & 98 & 8,10 & 0,86 & 10,61 & 1,12 & 3,22 & 39,75 & 1,66 \\
\hline
\end{tabular}


Tabla 1. Comparación de indicadores de impacto de Tasa de Mortalidad Infantil comunal en relación a RM y comuna referencial, 2005-2014 (continuación)

\begin{tabular}{|c|c|c|c|c|c|c|c|c|c|}
\hline Comuna & $\begin{array}{l}\text { Nacimientos } \\
\text { totales }\end{array}$ & $\begin{array}{l}\text { Defunciones } \\
\text { infantiles totales }\end{array}$ & $\begin{array}{c}\text { TMI } \\
\text { (por mil RNV) }\end{array}$ & RAP* & RAP\%* & $\mathrm{RT}^{*}$ & $\operatorname{RAPC} \wedge$ & $\mathrm{RAC} \% \wedge$ & $\mathrm{RT} \wedge$ \\
\hline San Miguel & 12.493 & 104 & 8,32 & 1,08 & 13,03 & 1,15 & 3,44 & 41,35 & 1,70 \\
\hline San Ramón & 15.107 & 100 & 6,62 & $-0,62$ & $-9,37$ & 0,91 & 1,74 & 26,28 & 1,36 \\
\hline Vitacura & 12.064 & 69 & 5,72 & $-1,52$ & $-26,58$ & 0,79 & 0,84 & 14,69 & 1,17 \\
\hline Total Prov. Santiago & 729.745 & 5.179 & 7,10 & $-0,14$ & $-2,01$ & 0,98 & 2,22 & 31,27 & 1,45 \\
\hline Puente Alto & 83.953 & 623 & 7,42 & 0,18 & 2,44 & 1,02 & 2,54 & 34,23 & 1,52 \\
\hline Pirque & 2.913 & 21 & 7,21 & $-0,03$ & $-0,43$ & 1,00 & 2,33 & 32,32 & 1,48 \\
\hline San José de Maipo & 1.944 & 10 & 5,14 & $-2,10$ & $-40,75$ & 0,71 & 0,26 & 5,06 & 1,05 \\
\hline Total Prov. Cordillera & 88.810 & 654 & 7,36 & 0,12 & 1,68 & 1,02 & 2,48 & 33,70 & 1,51 \\
\hline Colina & 19.584 & 150 & 7,66 & 0,42 & 5,47 & 1,06 & 2,78 & 36,29 & 1,57 \\
\hline Lampa & 12.573 & 63 & 5,01 & $-2,23$ & $-44,49$ & 0,69 & 0,13 & 2,59 & 1,03 \\
\hline Tiltil & 2.351 & 24 & 10,21 & 2,97 & 29,08 & 1,41 & 5,33 & 52,20 & 2,09 \\
\hline Total Prov. Chacabuco & 34.508 & 237 & 6,87 & $-0,37$ & $-5,42$ & 0,95 & 1,99 & 28,97 & 1,41 \\
\hline San Bernardo & 48.344 & 436 & 9,02 & 1,78 & 19,72 & 1,25 & 4,14 & 45,90 & 1,85 \\
\hline Buin & 12.678 & 119 & 9,39 & 2,15 & 22,87 & 1,30 & 4,51 & 48,03 & 1,92 \\
\hline Calera de Tango & 3.235 & 29 & 8,96 & 1,72 & 19,24 & 1,24 & 4,08 & 45,54 & 1,84 \\
\hline Paine & 9.022 & 76 & 8,42 & 1,18 & 14,05 & 1,16 & 3,54 & 42,04 & 1,73 \\
\hline Total Prov. Maipo & 73.279 & 660 & 9,01 & 1,77 & 19,62 & 1,24 & 4,13 & 45,84 & 1,85 \\
\hline Melipilla & 16.309 & 113 & 6,93 & $-0,31$ & $-4,49$ & 0,96 & 2,05 & 29,58 & 1,42 \\
\hline Alhué & 1.095 & 3 & 2,74 & $-4,50$ & $-164,26$ & 0,38 & $-2,14$ & $-78,10$ & 0,56 \\
\hline Curacaví & 3.851 & 26 & 6,75 & $-0,49$ & $-7,24$ & 0,93 & 1,87 & 27,70 & 1,38 \\
\hline María Pinto & 1.577 & 15 & 9,51 & 2,27 & 23,88 & 1,31 & 4,63 & 48,69 & 1,95 \\
\hline San Pedro & 1.006 & 4 & 3,98 & $-3,26$ & $-82,09$ & 0,55 & $-0,90$ & $-22,61$ & 0,82 \\
\hline Total Prov Melipilla & 23.838 & 161 & 6,75 & $-0,49$ & $-7,20$ & 0,93 & 1,87 & 27,70 & 1,38 \\
\hline Talagante & 10.101 & 67 & 6,63 & $-0,61$ & $-9,15$ & 0,92 & 1,75 & 26,40 & 1,36 \\
\hline El Monte & 4.770 & 33 & 6,92 & $-0,32$ & $-4,65$ & 0,96 & 2,04 & 29,48 & 1,42 \\
\hline Isla de Maipo & 4.654 & 37 & 7,95 & 0,71 & 8,93 & 1,10 & 3,07 & 38,62 & 1,63 \\
\hline Padre Hurtado & 7.021 & 54 & 7,69 & 0,45 & 5,87 & 1,06 & 2,81 & 36,54 & 1,58 \\
\hline Peñaflor & 12.447 & 82 & 6,59 & $-0,65$ & $-9,90$ & 0,91 & 1,71 & 25,95 & 1,35 \\
\hline Total Prov. Talagante & 38.993 & 273 & 7,00 & $-0,24$ & $-3,41$ & 0,97 & 2,12 & 30,29 & 1,43 \\
\hline
\end{tabular}

Sin considerar las comunas de Alhué y San Pedro, que cuentan con natalidades extremadamente bajas y defunciones excepcionales, Lo Barnechea, Vitacura, Las Condes, Nuñoa y Pudahuel mostraron tasas inferiores a 6 por mil NV. La comuna de Las Condes es la que presenta una menor TMI, siendo ésta de 4,88 defunciones por mil NV en el decenio. El RAP\% es de un $-48,4 \%$, con una RT de 0,67 (tabla 1 ).

\section{Comparación inter-comunal}

La comuna de Las Condes, al tener la menor TMI, se eligió como la comuna referencial. Al comparar las otras comunas con ella se observa que el RAPC\% de las comunas de la RM varía ampliamente, alcanzando un máximo de $61,6 \%$ en la comuna de Independencia, con una RT entre ambas comunas de 2,6 (tabla 1).

\section{Análisis de la mortalidad infantil tardía}

En el análisis específico de la TMIT por comunas Vitacura presenta la menor tasa, siendo ésta de 1,08 por mil RNV, mientras que la comuna de Independencia presenta la mayor tasa, la cual es de 4,38 por mil RNV. Al compararla con el estándar regional, el RAP de la comuna de Vitacura es de -1,05, el RAP\% -97,6 y 


\begin{tabular}{|c|c|c|c|c|c|c|c|c|c|}
\hline Comuna & $\begin{array}{l}\text { Nacimientos } \\
\text { totales }\end{array}$ & $\begin{array}{c}\text { Defunciones } \\
\text { infantiles tardías } \\
\text { totales }\end{array}$ & $\begin{array}{c}\text { TMIT } \\
\text { (por mil RNV) }\end{array}$ & RAP* & RAP\%* & $\mathrm{RT}^{*}$ & RAPC^ & $\mathrm{RAC} \% \wedge$ & $\mathrm{RT} \wedge$ \\
\hline Santiago & 44.574 & 69 & 1,55 & $-0,58$ & $-37,60$ & 0,73 & 0,47 & 30,23 & 1,43 \\
\hline Cerrillos & 11.711 & 25 & 2,13 & 0,00 & 0,22 & 1,00 & 1,05 & 49,41 & 1,98 \\
\hline Cerro Navia & 20.607 & 42 & 2,04 & $-0,09$ & $-4,51$ & 0,96 & 0,96 & 47,01 & 1,89 \\
\hline Conchalí & 18.635 & 49 & 2,63 & 0,50 & 18,99 & 1,23 & 1,55 & 58,93 & 2,43 \\
\hline El Bosque & 25.058 & 61 & 2,43 & 0,30 & 12,50 & 1,14 & 1,35 & 55,64 & 2,25 \\
\hline Estación Central & 17.931 & 41 & 2,29 & 0,16 & 6,85 & 1,07 & 1,21 & 52,77 & 2,12 \\
\hline Huechuraba & 15.808 & 38 & 2,40 & 0,27 & 11,39 & 1,13 & 1,32 & 55,07 & 2,23 \\
\hline Independencia*** & 11.657 & 51 & 4,38 & 2,25 & 51,31 & 2,05 & 3,30 & 75,31 & 4,05 \\
\hline La Cisterna & 11.002 & 22 & 2,00 & $-0,13$ & $-6,52$ & 0,94 & 0,92 & 45,99 & 1,85 \\
\hline La Florida & 48.214 & 87 & 1,80 & $-0,33$ & $-18,04$ & 0,85 & 0,72 & 40,15 & 1,67 \\
\hline La Granja & 19.699 & 41 & 2,08 & $-0,05$ & $-2,34$ & 0,98 & 1,00 & 48,11 & 1,93 \\
\hline La Pintana & 34.063 & 71 & 2,08 & $-0,05$ & $-2,19$ & 0,98 & 1,00 & 48,19 & 1,93 \\
\hline La Reina & 9.885 & 18 & 1,82 & $-0,31$ & $-16,97$ & 0,85 & 0,74 & 40,69 & 1,69 \\
\hline Las Condes & 36.282 & 44 & 1,21 & $-0,92$ & $-75,64$ & 0,57 & 0,13 & 10,94 & 1,12 \\
\hline Lo Barnechea & 12.826 & 22 & 1,72 & $-0,41$ & $-24,18$ & 0,81 & 0,64 & 37,04 & 1,59 \\
\hline Lo Espejo & 15.934 & 36 & 2,26 & 0,13 & 5,72 & 1,06 & 1,18 & 52,20 & 2,09 \\
\hline Lo Prado & 14.611 & 41 & 2,81 & 0,68 & 24,09 & 1,32 & 1,73 & 61,51 & 2,60 \\
\hline Macul & 15.190 & 27 & 1,78 & $-0,35$ & $-19,83$ & 0,83 & 0,70 & 39,24 & 1,65 \\
\hline Maipú & 70.809 & 132 & 1,86 & $-0,27$ & $-14,26$ & 0,88 & 0,78 & 42,07 & 1,73 \\
\hline Ñuñoa & 24.106 & 35 & 1,45 & $-0,68$ & $-46,70$ & 0,68 & 0,37 & 25,62 & 1,34 \\
\hline Pedro Aguirre Cerda & 14.564 & 34 & 2,33 & 0,20 & 8,76 & 1,10 & 1,25 & 53,74 & 2,16 \\
\hline Peñalolén & 38.315 & 86 & 2,24 & 0,11 & 5,10 & 1,05 & 1,16 & 51,88 & 2,08 \\
\hline Providencia & 17.984 & 42 & 2,34 & 0,21 & 8,80 & 1,10 & 1,26 & 53,76 & 2,16 \\
\hline Pudahuel & 35.091 & 70 & 1,99 & $-0,14$ & $-6,78$ & 0,94 & 0,91 & 45,86 & 1,85 \\
\hline Quilicura & 32.123 & 78 & 2,43 & 0,30 & 12,28 & 1,14 & 1,35 & 55,52 & 2,25 \\
\hline Quinta Normal & 15.075 & 43 & 2,85 & 0,72 & 25,33 & 1,34 & 1,77 & 62,14 & 2,64 \\
\hline Recoleta & 23.662 & 64 & 2,70 & 0,57 & 21,25 & 1,27 & 1,62 & 60,07 & 2,50 \\
\hline Renca & 22.565 & 57 & 2,53 & 0,40 & 15,68 & 1,19 & 1,45 & 57,25 & 2,34 \\
\hline San Joaquín & 12.100 & 23 & 1,90 & $-0,23$ & $-12,06$ & 0,89 & 0,82 & 43,18 & 1,76 \\
\hline San Miguel & 12.493 & 20 & 1,60 & $-0,53$ & $-33,05$ & 0,75 & 0,52 & 32,54 & 1,48 \\
\hline San Ramón & 15.107 & 23 & 1,52 & $-0,61$ & $-39,90$ & 0,71 & 0,44 & 29,06 & 1,41 \\
\hline Vitacura** & 12.064 & 13 & 1,08 & $-1,05$ & $-97,66$ & 0,51 & 0,00 & 0,00 & 1,00 \\
\hline Total Prov. Santiago & 729.745 & 1505 & 2,06 & $-0,07$ & $-3,40$ & 0,97 & 0,98 & 47,57 & 1,91 \\
\hline Puente Alto & 83.953 & 189 & 2,25 & 0,12 & 5,39 & 1,06 & 1,17 & 52,03 & 2,08 \\
\hline Pirque & 2.913 & 6 & 2,06 & $-0,07$ & $-3,41$ & 0,97 & 0,98 & 47,57 & 1,91 \\
\hline San José de Maipo & 1.944 & 3 & 1,54 & $-0,59$ & $-38,02$ & 0,72 & 0,46 & 30,02 & 1,43 \\
\hline Total Prov. Cordillera & 88.810 & 198 & 2,23 & 0,10 & 4,48 & 1,05 & 1,15 & 51,57 & 2,06 \\
\hline Colina & 19.584 & 50 & 2,55 & 0,42 & 16,57 & 1,20 & 1,47 & 57,70 & 2,36 \\
\hline Lampa & 12.573 & 22 & 1,75 & $-0,38$ & $-21,73$ & 0,82 & 0,67 & 38,28 & 1,62 \\
\hline Tiltil & 2.351 & 10 & 4,25 & 2,12 & 49,92 & 2,00 & 3,17 & 74,61 & 3,94 \\
\hline Total Prov. Chacabuco & 34.508 & 82 & 2,38 & 0,25 & 10,50 & 1,12 & 1,30 & 54,62 & 2,20 \\
\hline San Bernardo & 48.344 & 118 & 2,44 & 0,31 & 12,73 & 1,15 & 1,36 & 55,75 & 2,26 \\
\hline Buin & 12.678 & 39 & 3,08 & 0,95 & 30,76 & 1,44 & 2,00 & 64,89 & 2,85 \\
\hline Calera de Tango & 3.235 & 11 & 3,40 & 1,27 & 37,36 & 1,60 & 2,32 & 68,24 & 3,15 \\
\hline Paine & 9.022 & 29 & 3,21 & 1,08 & 33,73 & 1,51 & 2,13 & 66,40 & 2,98 \\
\hline Total Prov. Maipo & 73.279 & 197 & 2,69 & 0,56 & 20,82 & 1,26 & 1,61 & 59,85 & 2,49 \\
\hline
\end{tabular}


Tabla 2. Comparación de indicadores de impacto de Tasa de Mortalidad Infantil Tardía comunal en relación a RM y comuna referencial, 2005-2014 (continuación)

\begin{tabular}{|c|c|c|c|c|c|c|c|c|c|}
\hline Comuna & $\begin{array}{c}\text { Nacimientos } \\
\text { totales }\end{array}$ & $\begin{array}{c}\text { Defunciones } \\
\text { infantiles tardías } \\
\text { totales }\end{array}$ & $\begin{array}{c}\text { TMIT } \\
\text { (por mil RNV) }\end{array}$ & RAP* & RAP\%* & $R T^{*}$ & RAPC^ & $\operatorname{RAC} \% \wedge$ & $\mathrm{RT}^{\wedge}$ \\
\hline Melipilla & 16.309 & 34 & 2,08 & $-0,05$ & $-2,17$ & 0,98 & 1,00 & 48,19 & 1,93 \\
\hline Alhué & 1.095 & 0 & & . & & . & . & . & \\
\hline Curacaví & 3.851 & 8 & 2,08 & 0,00 & 0,00 & 0,98 & 0,00 & 0,00 & 1,92 \\
\hline María Pinto & 1.577 & 6 & 3,80 & 1,67 & 44,02 & 1,79 & 2,72 & 71,61 & 3,52 \\
\hline San Pedro & 1.006 & 2 & 1,99 & $-0,14$ & $-7,14$ & 0,93 & 0,91 & 45,68 & 1,84 \\
\hline Total Prov Melipilla & 23.838 & 50 & 2,10 & $-0,03$ & $-1,43$ & 0,99 & 1,02 & 48,57 & 1,94 \\
\hline Talagante & 10.101 & 14 & 1,39 & $-0,74$ & $-53,68$ & 0,65 & 0,31 & 22,08 & 1,28 \\
\hline El Monte & 4.770 & 6 & 1,26 & $-0,87$ & $-69,34$ & 0,59 & 0,18 & 14,14 & 1,16 \\
\hline Isla de Maipo & 4.654 & 11 & 2,36 & 0,23 & 9,88 & 1,11 & 1,28 & 54,31 & 2,19 \\
\hline Padre Hurtado & 7.021 & 16 & 2,28 & 0,15 & 6,53 & 1,07 & 1,20 & 52,61 & 2,11 \\
\hline Peñaflor & 12.447 & 28 & 2,25 & 0,12 & 5,31 & 1,06 & 1,17 & 51,99 & 2,08 \\
\hline Total Prov. Talagante & 38.993 & 75 & 1,92 & $-0,21$ & $-10,94$ & 0,90 & 0,84 & 43,75 & 1,78 \\
\hline
\end{tabular}

la RT 0,51, mientras que los indicadores de magnitud de la comuna de Independencia son RAP 2,25, RAP\% 51,31 y RT 2,05 (tabla 2).

En el análisis con la comuna de Vitacura como referencia, se observa una mayor variación que la observada en la TMI, alcanzando valores máximos en la comuna de Independencia. En esta comuna el RAPC es de 3,3 por mil RNV, el RAPC\% de 75,3\% y la RT de 4,05 (tabla 2).

\section{Evaluación de tendencia}

Se observó que la TMI de la RM sigue una tendencia a la disminución respecto al 2005 (coef. -0,075; IC95\%: - 0,11 a -0,03), lo que indica que la TMI disminuye en promedio por año 0,075 por mil NV (tabla $3)$. Las comunas que mostraron un aumento significativo en el periodo fueron Lo Barnechea (coef. 0,33; IC95\%: 0,07 a 0,58), Lo Espejo (coef. 0,27; IC95\%:0,05 a 0,49) y Recoleta (coef. 0,45; IC95\%:0,18 a 0,73). Las comunas que mostraron una disminución respecto el año 2005 fueron Las Condes (coef.-0,24; IC95\%: -0,41 a -0.07), Macul (coef. -0,31; IC95\%: -0,57 a -0,05), Pudahuel (coef. $-0,39$ (IC95\%: $-0,76$ a $-0,01$ ) y San Bernardo (coef. -0,23; IC95\%: -0,38 a -0,08) (figura 1). En cuanto a la TMIT, la RM presenta una tendencia negativa significativa en el decenio estudiado de 0,08 por mil NV (tabla 3). Peñalolén, Puente Alto, Las Con- des, Providencia, San Bernardo, Macul, Pudahuel, Talagante, Pedro Aguirre Cerda y Quilicura, presentaron disminuciones significativas en el decenio evaluado, mientras que solo Peñaflor presentó un aumento significativo en su TMIT (figura 2).

El mapa de calor muestra la distribución espacial de las TMI entre las RM, el color de la comuna corresponde a la magnitud del indicador. Cuanto más oscuro sea el color, mayor será el valor (figura 3).

\section{Discusión}

Los resultados del estudio muestran la persistencia de la heterogeneidad de la TMI entre las provincias y comunas de la RM; a pesar de la disminución significativa gracias a la implementación de políticas sociales, como lo es el Sistema de Protección Integral a la Infancia Chile Crece Contigo, y otras, se mantienen las inequidades. Nuestro estudio señala diferencias impensables en un país OCDE: el 43\% de las defunciones infantiles en menores de un año ocurridas en la comuna de Independencia podrían evitarse si ésta tuviera la misma TMI de la RM; el riesgo de presentar una defunción infantil es 75\% mayor en esta comuna que en la RM; el riesgo de haber fallecido en el primer año de vida en el decenio estudiado es de 2,6 veces por 
Tabla 3. Tendencia por comunas de la Región Metropolitana, 2005-2014

\begin{tabular}{|c|c|c|c|c|}
\hline \multirow[t]{2}{*}{ Población } & \multicolumn{2}{|l|}{ TMI } & \multicolumn{2}{|l|}{ TMIT } \\
\hline & Coef. (IC95\%)* & $\mathrm{p} \leq$ valor & Coef. (IC95\%)* & $\mathrm{p} \leq \mathrm{valor}$ \\
\hline$R M$ & $-0,075(-0,11$ a $-0,03)<$ & $0,005^{8}$ & $-0,08 \quad(-0,10$ a $-0,06)<$ & $0,001^{\&}$ \\
\hline Santiago & $-0,16 \quad(-0,36$ a 0,4$)$ & 0,107 & $-0,09 \quad(-0,19$ a 0,001$)$ & 0,053 \\
\hline Cerrillos & $0,01 \quad(-0,31$ a 0,33$)$ & 0,967 & $-0,18 \quad(-0,45$ a 0,09$)$ & 0,168 \\
\hline Cerro Navia & $-0,22 \quad(-0,59$ a 0,16$)$ & 0,223 & $-0,05 \quad(-0,18$ a 0,09$)$ & 0,420 \\
\hline Conchalí & $-0,14 \quad(-0,66$ a 0,38$)$ & 0,556 & $0,004(-0,34$ a 0,34$)$ & 0,976 \\
\hline El Bosque & $0,08 \quad(-0,49$ a 0,65$)$ & 0,753 & $-0,05 \quad(-0,26$ a 0,15$)$ & 0,550 \\
\hline Estación Central & $0,01 \quad(-0,50$ a 0,51$)$ & 0,970 & $-0,07 \quad(-0,45$ a 0,32$)$ & 0,697 \\
\hline Huechuraba & $0,05 \quad(-0,24$ a 0,35$)$ & 0,682 & $-0,03 \quad(-0,31$ a 0,25$)$ & 0,792 \\
\hline Independencia & $-0,20 \quad(-0,91$ a 0,51$)$ & 0,529 & $-0,07 \quad(-0,49$ a 0,36$)$ & 0,726 \\
\hline La Cisterna & $0,09 \quad(-0,28$ a 0,45$)$ & 0,599 & $0,16 \quad(-0,01$ a 0,33$)$ & 0,063 \\
\hline La Florida & $0,08 \quad(-0,20$ a 0,37$)$ & 0,515 & $0,07 \quad(-0,04$ a 0,19$)$ & 0,182 \\
\hline La Granja & $-0,11 \quad(-0,67$ a 0,45$)$ & 0,658 & $-0,18 \quad(-0,48$ a 0,12$)$ & 0,209 \\
\hline La Pintana & $-0,17 \quad(-0,37$ a 0,02$)$ & 0,077 & $-0,01 \quad(-0,13$ a 0,12$)$ & 0,890 \\
\hline La Reina & $0,17 \quad(-0,24$ a 0,57$)$ & 0,365 & $0,09 \quad(-0,03-$ a 0,22$)$ & 0,121 \\
\hline Las Condes & $-0,24 \quad(-0,41$ a $-0,07)<$ & $0,011^{\&}$ & $-0,21 \quad(-0,36$ a $-0,07)<$ & $0,010^{\&}$ \\
\hline Lo Barnechea & $0,33 \quad(0,07$ a 0,58$)>$ & $0,019 \&$ & $-0,29 \quad(-0,15$ a 0,74$)$ & 0,168 \\
\hline Lo Espejo & $0,27 \quad(0,05$ a 0,49$)>$ & $0,022^{8}$ & $-0,6 \quad(-0,17$ a 0,05$)$ & 0,247 \\
\hline Lo Prado & $-0,17 \quad(-0,42$ a 0,09$)$ & 0,170 & $-0,27 \quad(-0,55$ a 003$)$ & 0,052 \\
\hline Macul & $-0,31 \quad(-0,57$ a $-0,05)<$ & $0,025^{\&}$ & $-0,34 \quad(-0,55 a-0,14)<$ & $0,005^{8}$ \\
\hline Maipu & $-0,13 \quad(-0,31$ a 0,04$)$ & 0,109 & $-0,07 \quad(-, 021$ a 0,07$)$ & 0,290 \\
\hline Ñuñoa & $0,17 \quad(-0,13$ a 0,46$)$ & 0,226 & $0,04 \quad(-0,09$ a 0,17$)$ & 0,542 \\
\hline Pedro Aguirre Cerda & $-0,16 \quad(-0,72$ a 0,39$)$ & 0,520 & $-0,32 \quad(-0,58$ a $-0,06)<$ & $0,022^{\&}$ \\
\hline Peñalolén & $-0,07 \quad(-0,24$ a 0,09$)$ & 0,342 & $-0,16 \quad(-0,31$ a $-0,02)<$ & $0,029^{8}$ \\
\hline Providencia & $-0,43 \quad(-0,99$ a 0,13$)$ & 0,118 & $-0,22 \quad(-0,43$ a $-0,01)<$ & $0,039^{8}$ \\
\hline Pudahuel & $-0,39 \quad(-0,76$ a $-0,01)<$ & $0,044^{\&}$ & $-0,13 \quad(-0,21$ a $-0,05)<$ & $0,006^{8}$ \\
\hline Quilicura & $0,15 \quad(-0,37$ a 0,68$)$ & 0,523 & $-0,14 \quad(-0,19$ a $-0,09)<$ & $0,001^{\&}$ \\
\hline Quinta Normal & $0,13 \quad(-0,14$ a 0,40$)$ & 0,293 & $0,05 \quad(-0,21$ a 0,30$)$ & 0,691 \\
\hline Recoleta & $0,45 \quad(0,18$ a 0,73$)>$ & $0,005^{8}$ & $0,06 \quad(-0,14$ a 0,26$)$ & 0,536 \\
\hline Renca & $-0,01 \quad(-0,50$ a 0,47$)$ & 0,948 & $-0,16 \quad(-0,44$ a 0,12$)$ & 0,217 \\
\hline San Joaquín & $0,04 \quad(-0,44$ a 0,51$)$ & 0,866 & $-0,028(-0,28$ a 0,22$)$ & 0,802 \\
\hline San Miguel & $0,24 \quad(-0,14$ a 0,63$)$ & 0,184 & $0,10 \quad(-0,11$ a 0,31$)$ & 0,304 \\
\hline San Ramón & $0,46 \quad(-0,02$ a 0,93$)$ & 0,057 & $-0,003(-0,16-$ a 0,16$)$ & 0,958 \\
\hline Vitacura & $-0,46 \quad(-1,01$ a 0,10$)$ & 0,095 & $-0,14 \quad(-0,31$ a 0,03$)$ & 0,098 \\
\hline Puente Alto & $-0,22 \quad(-0,49$ a 0,05$)$ & 0,100 & $-0,17 \quad(-0,30$ a $-0,04)<$ & $0,015^{\&}$ \\
\hline Pirque & $-0,55 \quad(-2,02$ a 0,92$)$ & 0,411 & $-0,76 \quad(-1,38 a-0,14)$ & 0,023 \\
\hline San José del Maipo & $-0,02 \quad(-1,73$ a 1,70$)$ & 0,984 & $0,22 \quad(-0,39$ a 0,95$)$ & 0,373 \\
\hline Colina & $0,20 \quad(-0,23$ a 0,64$)$ & 0,316 & $-0,03 \quad(-0,19$ a 0,12$)$ & 0,661 \\
\hline Lampa & $-0,02 \quad(-0,40$ a 0,36$)$ & 0,902 & $-0,06 \quad(-0,26$ a 0,12$)$ & 0,418 \\
\hline Tiltil & $-0,72 \quad(-2,05$ a 0,60$)$ & 0,246 & $-0,46 \quad(-1,37$ a 0,44$)$ & 0,269 \\
\hline San Bernardo & $-0,23 \quad(-0,38$ a $-0,08)<$ & $0,007^{\&}$ & $-0,22 \quad(-0,34 a-0,10)<$ & $0,003^{8}$ \\
\hline Buin & $0,17 \quad(-0,95$ a 1,29$)$ & 0,736 & $0,14 \quad(-0,38$ a 0,67$)$ & 0,545 \\
\hline Calera de Tango & $-0,10 \quad(-1,38$ a 1,19$)$ & 0,863 & $-0,43 \quad(-0,12$ a 0,33$)$ & 0,230 \\
\hline Paine & $-0,27 \quad(-0,78$ a 0,23$)$ & 0,252 & $-0,22 \quad(-0,75$ a 0,30$)$ & 0,367 \\
\hline Melipilla & $-0,20 \quad(-0,65$ a 0,25$)$ & 0,341 & $0,09 \quad(-0,22$ a 0,39$)$ & 0,528 \\
\hline Alhué & $-0,15 \quad(-1,51$ a 1,21$)$ & 0,806 & - & - \\
\hline Curacaví & $-0,21 \quad(-1,88$ a 1,47$)$ & 0,785 & $0,31 \quad(-0,03$ a 0,64$)$ & 0,068 \\
\hline María Pinto & $-0,22 \quad(-0,91$ a 0,48$)$ & 0,495 & $0,50 \quad(-0,25$ a 1,26$)$ & 0,162 \\
\hline San Pedro & $-0,66 \quad(-1,96$ a 0,64$)$ & 0,276 & $-0,29 \quad(-0,1,16$ a 0,56$)$ & 0,445 \\
\hline Talagante & $-0,06 \quad(-0,56$ a 0,44$)$ & 0,785 & $-0,13 \quad(-0,23$ a $-0,03)<$ & $0,016^{8}$ \\
\hline El Monte & $-0,24 \quad(-0,98$ a 0,50$)$ & 0,477 & $-0,19 \quad(-0,41$ a 0,031$)$ & 0,084 \\
\hline Isla Maipo & $-0,45 \quad(-0,94$ a 0,05$)$ & 0,070 & $-0,33 \quad(-0,66-0,002)$ & 0,051 \\
\hline Padre Hurtado & $0,10 \quad(-1,02$ a 1,21$)$ & 0,844 & $0,17 \quad(-0,65-a) 0,98)$ & 0,643 \\
\hline Peñaflor & $0,21 \quad(-0,02$ a 0,44$)$ & 0,072 & $0,18 \quad(0,06$ a 0,31$)>$ & $0,009^{8}$ \\
\hline
\end{tabular}

*Intervalo de confianza al 95\%. ${ }^{8}$ Estadísticamente significativo. $>$ Aumenta. $<$ Disminuye 


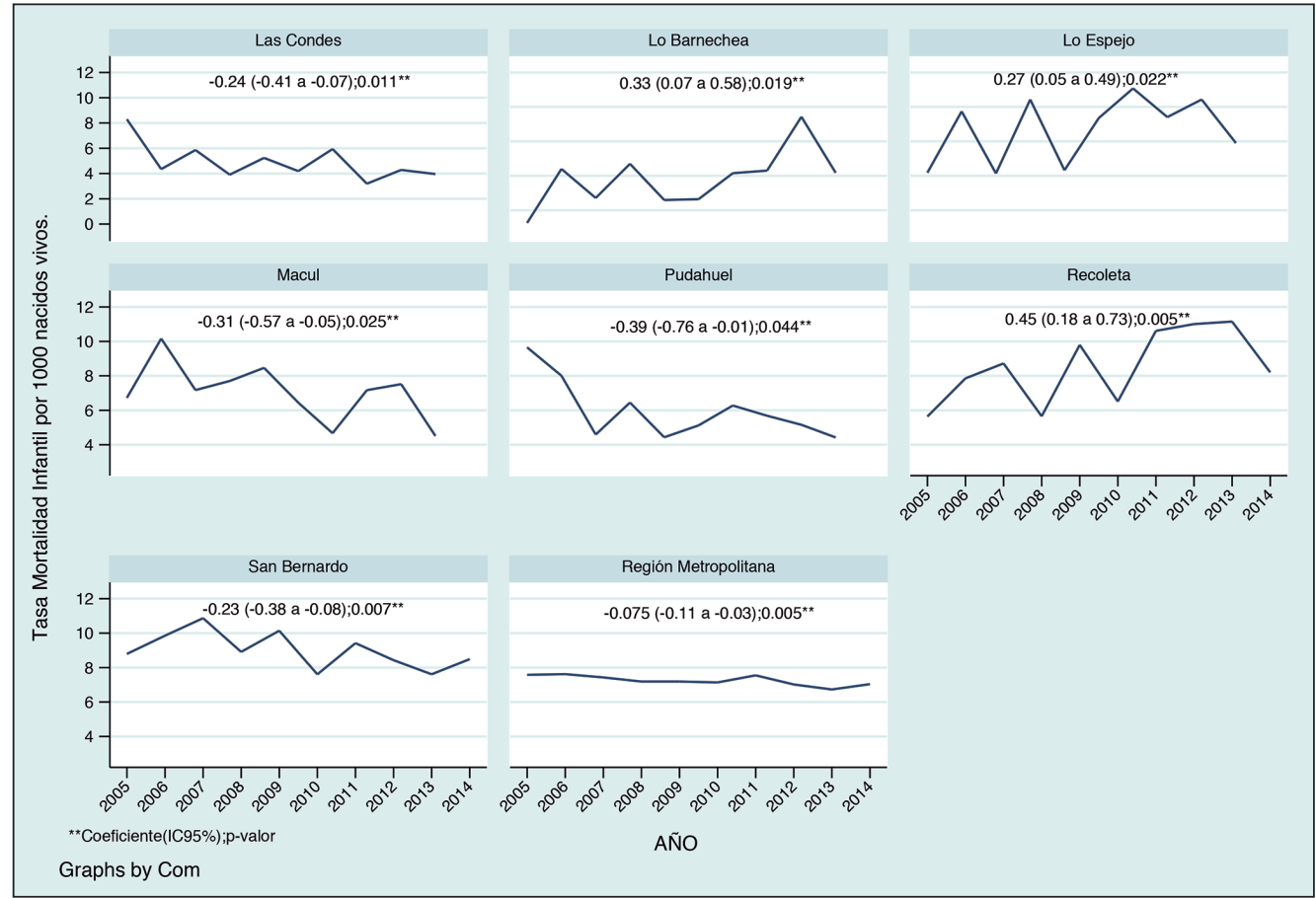

Figura 1. Comunas con cambios significativos en la tendencia de la Tasa de Mortalidad Infantil, Región Metropolitana, 2005-2014.

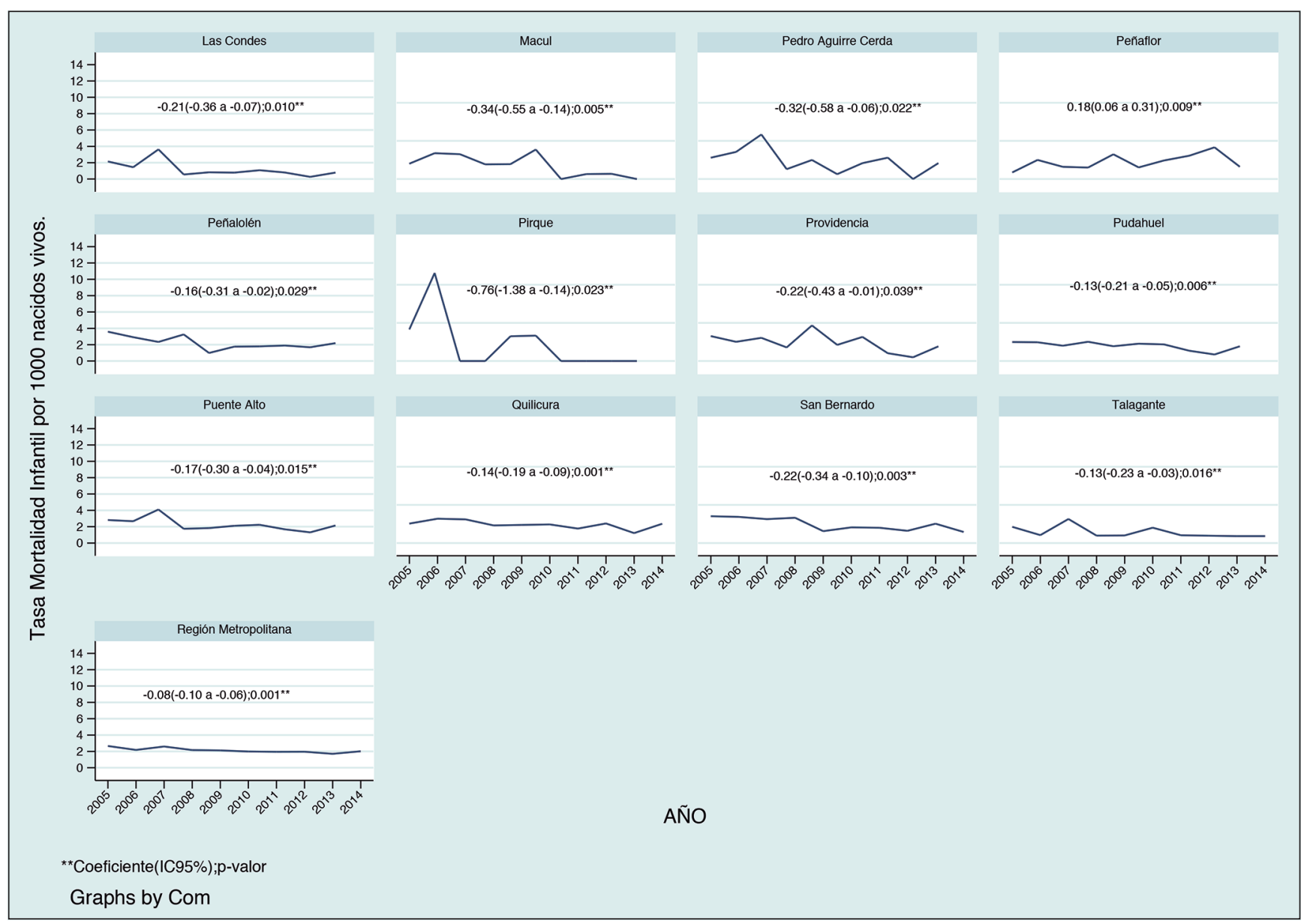

Figura 2. Comunas con cambios significativos en la tendencia de la Tasa de Mortalidad Infantil Tardía, Región Metropolitana, 2005-2014 


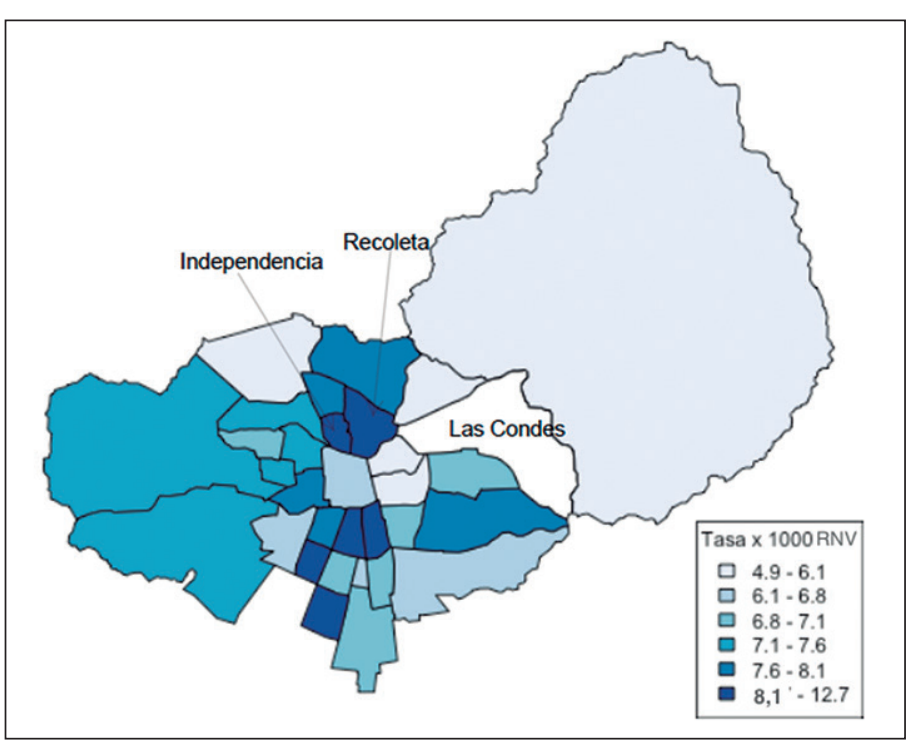

Figura 3. Mapa de calor de tasa de mortalidad infantil en comunas de Región Metropolitana, periodo 2005-2014

el solo hecho de haber nacido en Independencia, respecto del riesgo que tiene el niño nacido en la comuna de las Condes. En particular, al analizar la TMIT que agrupa principalmente las causas de muerte prevenibles de la primera infancia, la diferencia es aún mayor. Una de cada dos defunciones infantiles posteriores al mes de vida de la comuna de Independencia podrían evitarse si ésta tuviera los mismos indicadores de la $\mathrm{RM}$, mientras que el riesgo de haber fallecido entre el mes de vida y el año de vida en el decenio estudiado en Independencia es cuatro veces mayor al riesgo que tiene el niño cuya madre reside en la comuna de Vitacura. Estas diferencias podrían ser explicadas por los determinantes sociales de la salud que se ha demostrado que impactan en la mortalidad infantil, como el tabaquismo en el embarazo ${ }^{19}$, el menor nivel de escolaridad de la madre ${ }^{20} \mathrm{y}$ la falta de adherencia a controles e indicaciones médicas, los que son más prevalentes en grupos con privación social.

Las diferencias a nivel comunal podrían reflejar una segregación residencial que podría ser consistente con la disparidad de los indicadores sanitarios, económicos, sociales y ambientales entre comunas ${ }^{21}$. El área norte, donde se encuentra Independencia, tiene mayor porcentaje de hogares pobres, con mayor hacinamiento, mayor tasa de desocupación y un promedio de años de escolaridad inferior al promedio nacional y al de la $\mathrm{RM}^{22}$. A ello se suma que es una de las zonas que reúne un importante porcentaje de inmigrantes $(4,7 \%)$ provenientes de Perú, Haití, Argentina y Colombia ${ }^{29}$, situación que ha influenciado en el incremento de la natalidad especialmente en los últimos años, por las características de la población mayoritariamente fe- menina y joven, similar a lo descrito en otras regiones del mundo, lo que se ha denominado "feminización de la migración"23. El aumento de la TMI podría ser explicado por el aumento de la natalidad del grupo de madres inmigrantes de países que tienen bajas tasas de los controles de embarazo, o debido a las barreras que dificultan el acceso de esta población a los servicios de salud en nuestro país, como la falta de entrega oportuna de información, aspectos culturales, barreras idiomáticas, geográficas y administrativas que se acentúan en migrantes indocumentados ${ }^{24,25}$. En Chile existen estudios que evidencian que las mujeres inmigrantes, especialmente afrodescendientes, presentan escaso control del embarazo y, por lo tanto, pesquisa tardía de enfermedades maternas y fetales ${ }^{26}$. En los últimos años el Ministerio de Salud de Chile ha modificado el marco normativo con miras a mejorar el acceso a la atención de salud de los inmigrantes, en especial, de algunos grupos prioritarios, como protección de las embarazadas, atención de niños/as, atención de urgencia y atención a inmigrantes indocumentados. De esta manera, en la Circular A 15 N$^{\circ} 6$ establece que todos los establecimientos integrantes del Sistema Público de Salud deberán brindar tanto la atención como prestaciones en salud independiente de su situación jurídica en el país ${ }^{24}$. Sin embargo, estas normativas no siempre son de conocimiento de la población beneficiaria. Aun en este contexto de creciente inmigración, las brechas en las TMI a nivel comunal en nuestro país se mantienen de larga data ${ }^{27}$, por lo que la migración es solo un factor que acentuaría estas diferencias. En Chile la disminución de la TMI ocurrió en respuesta a intervenciones a nivel de salud pública ${ }^{20}$, sin embargo, aún sigue siendo un desafío una educación de calidad sin diferencias por nivel socioeconómico ${ }^{28}$. Así, en Chile comunas con altas TMI como Independencia y San Bernardo tienen porcentajes de pobreza de $9,8 \%$ y $9,2 \%$, respectivamente, mientras que en Las Condes y Vitacura estos porcentajes son de 0,6 y $0 \%{ }^{29}$.

Las diferencias registradas en tasas globales de mortalidad infantil, se reafirman también en la evaluación de las tendencias de la TMI por comuna, donde Lo Barnechea, Lo Espejo y Recoleta han incrementado sus tasas. En el caso de Lo Barnechea y Lo Espejo, podría explicarse por el crecimiento de las comunas en número de habitantes en los últimos años, generalmente a expensas de población joven. Recoleta, por su parte, alberga un importante número de inmigrantes que, como ya se mencionó, influye en la natalidad y mortalidad. Por otra parte, las comunas que disminuyeron la TMI en el periodo fueron Las Condes, Macul, $\mathrm{Pu}-$ dahuel y San Bernardo. La disminución de la TMI en Las Condes es consistente con los elevados indicadores socioeconómicos, de calidad de vida y el elevado número de población mayor de 60 años en la comuna 
(20,34\%). Al contrario, Macul, Pudahuel y San Bernardo mantienen niveles de pobreza superiores $(5,3 \%$, $7,8 \%$ y $9,2 \%$, respectivamente ${ }^{29}$.

La estrategia enfocada en intervenciones individuales para mejorar el nivel de salud y disminuir las inequidades no ha demostrado por sí sola ser efectiva a diferencia de las intervenciones comunitarias ${ }^{30}$. Por lo tanto, las estrategias deben tener un enfoque universal e intersectorial, más allá del sector salud. Ejemplo de una política exitosa de carácter universal que ha disminuido la brecha de inequidad de condiciones para el desarrollo infantil, ha sido el mayor tiempo libre parental remunerado para asistir a citas prenatales y para el cuidado en los primeros meses de vida, tanto de la madre como del padre ${ }^{31}$. Esta medida se ha materializado en Chile con la ampliación del tiempo postnatal parental. Las mayores reducciones en la TMI en nuestro país se relacionaron con las mejoras en las condiciones de vida en términos de vivienda y educación, al aumento en el gasto en salud, y la implementación y mejoramiento de programas de salud maternoinfantil, destacando los programas de alimentación complementaria, el control prenatal y control del niño sano ${ }^{32,33}$. Hoy en día, la existencia de programas específicos que educan y lograr un cambio en las conductas de riesgo en grupos sociales menos favorecidos ${ }^{34}$ como ha sido el programa IRA en Chile, política universal y que cuenta con estrategias específicas para pacientes con sindrome bronquial obstructivo crónico o asma ${ }^{35}$, permitirán mantener las reducciones en las TMI alcanzadas hoy. Sin embargo, persiste la dificultad para reducir aún más los ya bajos niveles de TMI, dado que los principales contribuyentes a ella son la prematurez y las malformaciones congénitas, los que son difíciles de prevenir por su origen multicausal. El aumento de las comorbilidades crónicas durante el embarazo, de la edad gestacional y los tratamientos de fertilidad con una mayor probabilidad de embarazo múltiple, son, entre otros, factores de riesgo demostrados en población chilena, que contribuyen a aumentar la tasa de partos prematuros. Sin embargo, se han identificado estrategias concretas para reducir la mortalidad asociada a prematurez, donde el acceso a una salud equitativa $\mathrm{y}$ de calidad juega un rol fundamental ${ }^{36}$. En Chile, este escenario se suma al de la 'la tiranía de los promedios' que oculta los fenómenos aun presentes, representados en las inequidades entre comunas no resueltas como las presentadas en este trabajo, y a lo que se suman desafíos futuros, como aquellos derivados de la migración, la transculturalidad y el desarrollo propio de un país que podría contribuir a incrementar la tasa de partos prematuros. Ello supone que, aquellas estrategias que fueron efectivas para reducir la TMI en grupos de personas con mayores niveles de pobreza y menor nivel de educación, deberán sumarse a aquellas propias de la transición epidemiológica de nuestro país.

En los años 60 el problema de la TMI fue abordado desde una perspectiva mucho más amplia que la que correspondía al sector salud, considerando el binomio madre-hijo y los determinantes sociales en donde éste se desarrollaba. Hoy, cuando tenemos mejores registros y herramientas metodológicas más precisas y asequibles, debemos retomar el análisis de la situación de TMI y, con una mirada preventiva, proponer medidas consistentes para mantener los logros alcanzados, reducir las inequidades dentro de nuestro país y evitar aumentos en la TMI en grupos de riesgo, por barreras de acceso que puedan enfrentar personas que se integran a nuestro sistema de salud. Asimismo, las estrategias tendientes a reducir los factores de riesgos de enfermedades no transmisibles permitirán mejorar la salud de las futuras embarazadas y su descendencia. Desde ese punto de vista, es fundamental concentrar esfuerzos intersectoriales en educación por medio de la mejora en su calidad, así como a nivel del sector salud por medio del fortalecimiento en la formación del equipo de salud en materias atingentes a las poblaciones de mayor riesgo, incorporando, por ejemplo, aspectos relativos a migración, transculturalidad y embarazo adolescente desde los primeros años de formación profesional y/o capacitando a los equipos de salud continuamente en estos aspectos, así como en los problemas de salud que hoy prevalenen en nuestra población. Asimismo, es fundamental abrir espacios de mayor participación a poblaciones vulnerables para la toma de decisiones en salud, lo que nos permita comprender por qué las políticas dirigidas a reducir las inequidades dentro de Chile no han sido suficientemente efectivas y nos lleve a repensar cuáles son los cambios de nuestra sociedad que hoy no están siendo suficientemente abordados para contribuir a la reducción de inequidades dentro de nuestro territorio, expresadas en la desigualdad en las TMI.

Limitaciones: Si bien en Chile la cobertura de las estadísticas de eventos vitales desde 1990 se considera robusta, una posible limitación de nuestro estudio se relaciona con la potencial variabilidad intercomunal en la calidad de las estadísticas, tal como se observa a nivel interregional, donde las muertes mal definidas varían entre $1,5 \%$ y $13,7 \%{ }^{37}$. Sin embargo, en Chile, la legislación vigente resguarda la calidad de las estadísticas vitales, a través de las auditorías de mortalidad infantil, mediante una normativa vigente y revisada en $2012^{38}$. Adicionalmente, en nuestro país la obligación legal de inscribir los nacimientos y defunciones data de 1885 , a lo que se suma la estimación y corrección por parte del INE del registro tardío de los nacimientos ${ }^{39}$.

En definitiva, este estudio evidencia que observar el indicador promedio regional oculta las diferencias de las TMI/TMIT existentes en el territorio. De ahí 
que las políticas públicas deben abordarse desde distintos planos y bajo la consigna "salud en todas las políticas", destacando los determinantes sociales expresados como marginación, pobreza, ruralidad y falta de acceso, y factores esenciales como educación y salud, que en diversos estudios han mostrado una estrecha relación con las TMI/TMIT, más aún en el escenario demográfico y epidemiológico actual, donde es un desafío identificar características del ambiente social y físico que pueden explicar estas inequidades, tales como migraciones y embarazo en edades extremas - embarazos de alto riesgo, que pueden conducir a cambios en el perfil de mortalidad infantil del país.

\section{Responsabilidades Éticas}

Protección de personas y animales: Los autores declaran que los procedimientos seguidos se conformaron a las normas éticas del comité de experimentación humana responsable y de acuerdo con la Asociación Médica Mundial y la Declaración de Helsinki.

Confidencialidad de los datos: Los autores declaran que han seguido los protocolos de su centro de trabajo sobre la publicación de datos de pacientes.

Derecho a la Privacidad y Consentimiento Informado: Los autores declaran que la información ha sido obtenida de datos anonimizados previamente, por lo cual el Comité de Ética de Investigación en uso de sus facultades, ha eximido de la obtención de un consentimiento informado, lo cual consta en el acta respectiva.

\section{Conflicto de intereses}

Los autores declaran no tener conflicto de intereses.

\section{Referencias}

1. Starfield B. Equity and health: a perspective on nonrandom distribution of health in the population. Rev Panam Salud Publica. 2002;12(6):384-7.

2. Harper S, Lynch J. Measuring health Inequalities, in. In: Oakes J, Kaufman J, editors. Methods in Social Epidemiology. San Francisco: John Wiley and Sons; 2006.

3. OECD Organization for Economic Co-operation and. Infant mortality rates in OCDE countries 2014 [Internet]. Available from: https://data.oecd.org/ healthstat/infant-mortality-rates.htm

4. Whitehead M. The Concepts and Principles of Equity and Health. Int J Heal Serv [Internet]. 1992;22(3):429-45. Available from: http://journals.sagepub. com/doi/10.2190/986L-LHQ6-2VTEYRRN

5. World Health Organization. Equity in health and health care: a WHO/ SIDA initiative [Internet]. World Heal. Geneva; 1996. Available from: http://apps.who.ihttp//apps.who. int/iris/bitstream/10665/63119/1/ WHO_ARA_96.1.pdf?ua=1nt/iris/ handle/10665/63119.

6. Braveman P, Starfield B, Geiger HJ. World Health Report 2000: how it removes equity from the agenda for public health monitoring and policy. BMJ Br Med J. 2001;323(september):678-81.

7. Illanes JP. [Mortality as an index of social development]. Estud Publicos [Internet]. 1984;16(16):73-116. Available from: http://www.ncbi.nlm.nih.gov/ pubmed/12267392.
8. Sen A. Mortality as an indicator of economic success and failure. Econ J. 1998;108(446):1-25.

9. Azria E. Inégalités sociales en santé périnatale. Arch Pediatr. 2015;22(10):1078-85.

10. Gover M. The Birth Rate and Infant and Maternal Mortality. Public Heal Rep. 61(43):1529-38.

11. Norman P, Gregory I, Dorling D, Baker A. Geographical trends ininfant mortality: England and Wales, 1970-2006. Health Statistics Q40:18-29, 2008. Heal Stat Q. 2008;40(Winter):18-29.

12. Casas JA, Dachs JNW, Bambas A. Health Disparities in Latin America and the Caribbean: The Role of Social and Economic Determinants. Pan Am Health. 1993;35(2):143-5.

13. Bahr J, Wehrhahn R. Life expectancy and Infant Mortality Rate in Latin America. 1993;36:1373-82.

14. Gonzalez R. Reduction in Neonatal Mortality in Chile Between 1990 and 2000. Pediatrics [Internet]. 2006;117(5):e949-54. Available from: http://pediatrics.aappublications.org/cgi/ doi/10.1542/peds.2005-2354

15. Donoso E. Inequalities in infant mortality in Santiago. Rev Med Chil. 2004;132(4):461-6.

16. Szot J. Caracterización de la Mortalidad Infantil Tardía en Chile, 1999. Rev Pediatría al día. 2002;18:79-82.

17. Kawachi I, Berkman L. Neighborhoods and Health. Press OU, editor. Oxford; 2003.

18. Kramer M, Hogue C. What Causes Racial Disparities in Very Preterm Birth? A
Biosocial Perspective. Epidemiol Rev. 2009;51(1):87-100.

19. Gray R, Bonellie SR, Chalmers J, et al. Contribution of smoking during pregnancy to inequalities in stillbirth and infant death in Scotland 1994-2003: Retrospective population based study using hospital maternity records. BMJ. 2009;339(7726):906-8.

20. Medina M, Cerda J. Nivel de Educación Parental y Mortalidad Infantil. Rev Chil Pediatr. 2010;81(3):228-33.

21. Schulz AJ, Williams DR, Israel BA, Lempert LB. Racial and Spatial Relations as Fundamental Determinants of Health in Detroit. Milbank Q. 2002;80(4):677-707.

22. Doña C, Moya C. Migración, ciudad y desigualdad en el Gran Santiago, Observatorio Desigualdades. 2017.

23. Pérez CD. Inmigración y fecundidad en España. Cuad de Geogr 2004;76:239-50.

24. MINSAL. Política de salud de migrantes internacionales. 2010.

25. Vásquez-de Kartzow R, Castillo-Durán C. Embarazo y parto en madres inmigrantes en Santiago, Chile. Rev Chil Pediatr. 2012;83(4):366-70.

26. Sánchez K, Valderas J, Messenger K, Sánchez C, Barrera F. Haití , la nueva comunidad inmigrante en Chile Haití. Rev Chil Pediatr. 2018;89(2):278-83.

27. Flores M, Cerda J. Evolución de la Desigualdad en la Mortalidad Infantil. Rev Chil Pediatr. 2010;81(3):215-20.

28. Riveros Cornejo LA, Báez Castillo GA. Chile y la OCDE. La dicotomia entre lo macroeconómico y el desarrollo humano. Estud Int. 2014;177:9-34. 
29. Ministerio De Desarrollo Social. CASEN 2015. 2015.

30. The Q, Of C, Context R. NIH Public Access. Soc Sci. 2009;67(8):1258-68.

31. Hollowell J, Kurinczuk JJ, Brocklehurst P, Gray R. Social and Ethnic Inequalities in Infant Mortality: A Perspective from the United Kingdom. Semin Perinatol [Internet]. 2011;35(4):240-4. Available from: http://dx.doi.org/10.1053/j. semperi.2011.02.021.

32. Kaempffer A, Medina E. Mortalidad infantil reciente en Chile: Éxitos y desafíos. Rev Chil Pediatr. 2006;77(5):492-500.
33. Szot J. Reseña de la salud pública materno-infantil chilena durante los últimos 40 años: 1960-2000. Rev Chil Obstet Ginecol. 2002;67(2):12935 .

34. Mackenbach JP, Bakker MJ. Tackling socioeconomic inequalities in health: Analysis of European experiences. Lancet. 2003;362(9393):1409-14

35. Girardi G, Astudillo P, Zúñiga F. El programa IRA en Chile: hitos e historia. Rev Chil Pediatría. 2001;72(4):292-300.

36. Mendoza Tascón LA, Claros Benítez DI, Mendoza Tascón LI, Arias Guatibonza MD, Peñaranda Ospina CB.
Epidemiología de la prematuridad, sus determinantes y prevención del parto prematuro. Rev Chil Obstet Ginecol. 2016;81(4):330-42.

37. Núñez F ML, Icaza N MG. Calidad de las estadísticas de mortalidad en Chile, 1997-2003. Rev Med Chile. 2006;134(9):1191-6.

38. Salud $\mathrm{M}$ de. Resolución exenta $\mathrm{N}^{\circ} 470$ que aprueba Norma General Técnica N ${ }^{\circ} 100$, "Norma y procedimientos para el registro de las auditorías de muertes maternas, fetales e infantiles." 2012.

39. Instituto Nacional de Estadísticas. Anuario de Estadísticas Vitales. 2016. 